\title{
EVALUATION OF NEURAL NETWORK COMPRESSION METHODS ON THE RESPIRATORY SOUND DATASET
}

\author{
Tamás Pál ${ }^{1}$, Bálint Molnár ${ }^{1}$, Ádám Tarcsi² and Csongor László Martin ${ }^{2}$ \\ ${ }^{1}$ Department of Information Systems, Faculty of Informatics, Eötvös Loránd University, Hungary \\ ${ }^{2}$ Department of Data Science, Faculty of Informatics, Eötvös Loránd University, Hungary
}

\begin{abstract}
Recently, the use of smart medical solutions has experienced significant growth and the area of Internet of Medical Things (IoMT) has been established as an independent field. Artificial intelligence-based analysis of physiological signal data has resulted in promising results. This paper aims to assess neural network compression possibilities applied to a respiratory classification problem. The experiment is carried out on an Nvidia Jetson TX2 edge device and a personal computer. Respiratory sounds are classified into 8 classes of disease using two distinct deep learning networks. The trained models are compressed using half-precision and 8-bit integer quantization methods, and the inference results are compared and analyzed based on predictive powers, memory footprint, and inference time. Traditional, interpretable models (SVM, KNN) are also compared to the former deep models. The results are promising, the compression techniques manage to decrease memory usage 8 times while experiencing a negligible decrease in model accuracy.
\end{abstract}

\section{KEYWORDS}

Model Compression, Respiratory Classification, IoT, Deep Learning

\section{INTRODUCTION}

The gradual automation of healthcare has allowed the expansion of embedded and wearable devices in the field. These smart devices coupled with artificial intelligence software can potentially lead to reliable, clinically acceptable solutions.

In the last few years, neural networks have established themselves as models with high predictive power and great generalization capability. A number of models have been developed for complex problems in computer vision or natural language processing that despite their near-perfect performance, have a huge number of layers that make their deployment on under-powered devices impossible. Thus, their complex and computationally expensive nature have resulted in limited applications on smart devices and IoT. This motivates the use of model compression, in which recent advances (quantization, pruning, low-rank approximation, knowledge distillation, weight sharing, etc...) have opened the gate for more widespread use of deep learning in IoT.

Established methods in feature extraction and machine learning for signal and audio classification can be applied to IoT devices using model compression. In this experiment, two deep learning architectures are submitted to half-precision (16-bit float weights) and 8-bit quantization (8-bit integer weights) experiments on an Nvidia Jetson TX2 and a PC. The developed solutions are applied to a respiratory sound dataset in the hope of achieving more efficient, more computational-friendly method combinations.

The detection of respiratory diseases has been an important field of study, as such illnesses are responsible for millions of deaths yearly. The Respiratory Sound database (Rocha, et al., 2018) used in this experiment is detailed in Section 3.2.

The main contributions of this paper are the positive results on the compressed models that indicate the presence of potential in the use of lightweight models operating on smart devices that tackle medical problems, like respiratory classification. 
Section 2 describes related research in neural network compression on edge devices. Sections 3.1, 3.3, 3.4 present the details of the experiment, the feature-extraction steps and the deep-learning models, respectively. The low-precision inference techniques are introduced in Section 3.5. The evaluation methodology and the results are presented in Sections 3.6 and 4, respectively.

\section{RELATED WORK}

Hadidi et al. (2019) evaluate the deployment possibilities of different deep learning frameworks on edge devices through the running/inference of different well-known models (ResNet, VGG, MobileNet, AlexNet) on a set of IoT/edge devices. The following frameworks were tested: TensorFlow, TensorFlow-Lite, Keras, Caffe/2, Movidius NCSDK Toolkit, PyTorch, TensorRT, DarkNet, FPGA Framework. The devices were the following: Raspberry Pi 3B, Nvidia Jetson TX2, and Nvidia Jetson Nano. The paper discusses the quantization and other model optimization capabilities of the frameworks in question.

Jain et al. (2020) propose a new dialect called QNN (Quantized Neural Network) which can be built into the deep learning compilers to support quantization operations across multiple platforms. They hope to fill the void caused by the lack of quantization support in some frameworks and also hardware configurations. The proposed solution is then tested on multiple devices of different architecture: Intel Xeon Cascade Lake CPUs, Nvidia Tesla T4 GPUs, ARM Cortex-A CPUs, and Raspberry Pi3 and Pi4. Acharya \& Basu (2020) consider the ICBHI challenge by downsampling to $4 \mathrm{kHz}$, extracting Mel-spectrograms, and using a hybrid CNN-RNN (Convolutional Neural Network - Recurrent Neural Network) model that can handle variable-sized input. The Int. Conf. on Biomedical Health Informatics (ICBHI) challenge consists of the task of classifying respiratory sounds into crackles and wheezes. The authors discuss the applicability of models in mobile systems and consider weight quantization as a tool for reducing the model complexity.

Yang et al. (2018) experiment with complex quantized convolutional neural networks on an Intel Edison IoT board. They propose a new, local-based quantization scheme. They compare the 32-bit floating-point inference with the 8-bit fixed point, 6-bit, 4-bit, and 2-bit fixed-point quantizations on two CNNs: AlexNet and VGG-16. The quantized networks' inference times were reduced to half of the 32-bit float network's time. Moreover, there was no significant drop in accuracy for the 8,6, and 4-bit quantization, while the 2-bit network produced results half as poor.

\section{METHODS}

\subsection{Experimental Design}

The experiment was designed in the following way: the models were constructed, trained, compressed and tested on the respective device. A detailed platform taxonomy of the experiment is presented in Table 1.

Table 1. Detailed Platform Taxonomy

\begin{tabular}{|l|l|l|l|}
\hline \multicolumn{4}{|l|}{ Information system: PC + Nvidia Jetson TX2 model training and compression experiment } \\
\hline Platform service category & Subcategory & Required & Supporting technology \\
\hline $\begin{array}{l}\text { Data Interchange } \\
\text { Services }\end{array}$ & Audio Processing & yes & $\begin{array}{l}\text { Librosa (resampling, splitting, MFCC } \\
\text { conversion) }\end{array}$ \\
\hline
\end{tabular}




\begin{tabular}{|l|l|l|l|}
\hline & $\begin{array}{l}\text { Electronic Data } \\
\text { Interchange }\end{array}$ & yes & Google Drive \\
\hline $\begin{array}{l}\text { Data Management } \\
\text { Services }\end{array}$ & $\begin{array}{l}\text { Data } \\
\text { Dictionary/Repository }\end{array}$ & yes & $\begin{array}{l}\text { Numpy (encode dataset into binary } \\
\text { format, .npy files) }\end{array}$ \\
\hline & Filtering & $\begin{array}{l}\text { Joblib (sklearn model persistence), } \\
\text { PyTorch (pickle-based model } \\
\text { serialization, .pt files), ONNX }\end{array}$ \\
\hline $\begin{array}{l}\text { Location and Directory } \\
\text { Services }\end{array}$ & Data Communications & yes & $\begin{array}{l}\text { Imbalanced-learn (undersampling of } \\
\text { majority class) }\end{array}$ \\
\hline Network Services & Distributed Data & yes & Google Drive \\
\hline $\begin{array}{l}\text { System and Network } \\
\text { Management Services }\end{array}$ & Software Installation & yes & pip (Python package manager system) \\
\hline $\begin{array}{l}\text { Operating System } \\
\text { Services }\end{array}$ & $\begin{array}{l}\text { Command Interpreter } \\
\text { and Utility }\end{array}$ & yes & $\begin{array}{l}\text { Nvidia SDK Manager } \\
\text { library) }\end{array}$ \\
\hline & $\begin{array}{l}\text { pror memory queries. Python } \\
\text { time (Python library) }\end{array}$ \\
\hline $\begin{array}{l}\text { Software Engineering } \\
\text { Services }\end{array}$ & $\begin{array}{l}\text { Programming } \\
\text { Language }\end{array}$ & yes & Python (3.6.9) \\
\hline & $\begin{array}{l}\text { Computer-Aided } \\
\text { Software Engineering } \\
\text { (CASE) Environment } \\
\text { and Tools }\end{array}$ & yes & Geany (IDE) \\
\hline Software library & yes & $\begin{array}{l}\text { PyTorch (1.6.0), Scikit-learn, TensorRT } \\
\text { (7.2.3.4), Neural Network Distiller }\end{array}$ \\
\hline
\end{tabular}

The neural network models were constructed using the PyTorch (Paszke, et al., 2019) deep learning framework. The dataset was saved to Numpy binaries and then transferred to the Jetson for the inference task.

The Nvidia Jetson TX2 module is a high-performance computing device, that is created to handle intensive AI tasks despite its small size. The device is comprised of a 256-core Nvidia Pascal GPU, a dual-core NVIDIA Denver2, and a Quad-Core ARM Cortex-A57 CPU, 8GB of memory, and 32 GB of storage space. The Jetson TX2's Pascal GPU architecture doesn't support 8-bit integer inference on the GPU but supports 16-bit float inference. The ARMv8-A architecture CPUs on the device support 8-bit integer quantization operations. The Jetson device has been supplied with the required drivers and libraries (CUDA, cuDNN, TensorRT) using the Nvidia SDK Manager software.

The installation of PyTorch on the Jetson is necessary to run the inference and quantization experiments. The framework currently only supports CPU-based quantization. Quantization operations are implemented using two back-end libraries: FBGEMM (for x86 systems) and QNNPACK (for ARM architectures). QNNPACK (Quantized Neural Network Package) provides implementations of common operations/layers in neural networks on quantized 8-bit tensors. Layers like 2D convolution, 2D max pooling, fully connected layer, activations are all supported. Unfortunately, the pre-built PyTorch wheels offered by Nvidia for the Jetson TX2 are built using the USE_QNNPACK=0 flag, thus QNNPACK will not be present in the installation. As the quantization operations of the library rely on QNNPACK, it is necessary to build PyTorch from the source with the proper build options.

The PC environment used for the other half of the experiments is equipped with an Intel(R) Core (TM) i5-10300H 2.50GHz CPU and an Nvidia GeForce GTX 1650 Mobile GPU. The same version of PyTorch and TensorRT is present with enabled quantization. 


\subsection{Dataset}

The Respiratory Sound Database (Rocha, et al., 2018) was created by a Portuguese and Greek research group. It is a set of 920 annotated breathing sounds containing 8 classes of recordings: 7 classes that indicate the presence of a specific disease and the class of healthy breathings. The data is collected from 126 patients that span all age groups: children, adults, elderly. Each recording is a collection of multiple consecutive breathing cycles, 6898 in total for the whole dataset. The dataset contains both clean and noisy recordings.

The recordings are from 7 locations on the upper body: trachea, left and right side of the anterior, posterior and lateral side of the chest. Single- and multi-channel acquisition mode is used.

The dataset has an imbalanced nature in terms of label distribution: the majority class (COPD - chronic obstructive pulmonary disease) contains 793 recordings, while Asthma has only one associated audio file. The lengths of the breathing cycles are also varied.

The recordings were captured by 3 different devices, therefore multiple sampling rates are present in the data: $4000 \mathrm{~Hz}, 10000 \mathrm{~Hz}, 44100 \mathrm{~Hz}$.

\subsection{Preprocessing and Feature Extraction}

Firstly, all recordings have been resampled to the common sampling frequency of $16000 \mathrm{~Hz}$. No filtering was applied. Then, the breathing cycles were extracted from the audio files. These recordings have been split into segments of 0.5 seconds with 0.125 second overlap. This resulted in an increase in the model performance. The final preprocessing step was to apply random undersampling, which reduced the imbalanced nature of the dataset.

The feature extraction step consists of extracting MFCC (Mel Frequency Cepstral Coefficients) (Mermelstein, 1976) spectrograms. MFCCs are intensively used in audio classification tasks since their introduction by Paul Mermelstein in 1976.

Algorithm:

1. Take the Short-time Fourier transform of the signal.

2. Apply the Mel filterbank to the power spectra of each frame, summing the energies in each frequency band. The Mel filterbank is a set of overlapping triangular filters.

3. Take the logarithm of the calculated energies.

4. Take the Discrete Cosine Transform (DCT) of the log filterbank energies, resulting in a so-called cepstrum. Intuitively, the cepstrum captures information on the rate of change in frequency bands.

5. The MFCC coefficients are the resulting DCT coefficients.

40 MFCC coefficients were used. Moreover, each coefficient was extended with its delta value (change in coefficient).

\subsection{Models}

\subsubsection{Dense Neural Network (DNN)}

This procedure involves the extraction of MFCC coefficients, reshaping of feature set through aggregation along the time axis, and finally feeding these to a dense neural network (DNN) classifier.

The peculiarity of this method lies in the reshaping step, which is described more clearly below. By calculating some statistical features across all frames, the spectrogram can be reduced to one feature vector, where statistical values (e.g.: mean + standard deviation) calculated for each frequency band are stacked vertically in a continuous fashion. Using this method, a feature set of shape $(k, n)$ will take the $(2 \times k, 1)$ form after the reshaping operation (Pál, Molnár, \& Tarcsi, 2020). This operation is illustrated in Equation 1 ( $\mu_{X}$ - mean of random variable, $\sigma_{X}$ - standard deviation of random variable). The reshaped dataset is then normalized with standard scaling. 


$$
\left[\begin{array}{cccc}
f_{11} & f_{12} & f_{13} & \ldots \\
f_{21} & f_{22} & f_{23} & \ldots \\
\vdots & & & \ddots
\end{array}\right] \Rightarrow\left[\begin{array}{c}
\mu_{f 1} \\
\sigma_{f 1} \\
\mu_{f 2} \\
\sigma_{f 2} \\
\vdots
\end{array}\right]
$$

Equation 1

The DNN classifier contains two hidden layers. The size of the first hidden layer is $1 / 2 \times(I+C)$, where $I$ is the size of the input and $C$ is the number of classes, thus having several nodes corresponding to the arithmetical mean of the input and output dimensionality. The second layer has $1 / 4 \times(I+3 \times C)$ nodes, which is the mean of the first hidden and output layer sizes. ReLU activations are used in the hidden layers, while Softmax activation is used in the output layer. This design is the result of testing multiple network sizes and topologies.

The DNN model has a very low number of trainable parameters, just 17,810 . The relatively low number of parameters is an advantage for use in IoT devices. For comparison, the CNN model presented below has 368,684 trainable parameters.

\subsubsection{D Convolutional Neural Network (CNN)}

This is the most widely used method for such tasks, it is used by feeding the MFCC spectrograms directly into a convolutional neural network $(\mathrm{CNN})$.

In terms of normalization, "per-image" normalization achieved the best results. The following transformation is applied to each spectrogram:

$$
X=\frac{X-\mu(X)}{\sigma(X)}
$$
$X$.

where $X$ marks a spectrogram matrix, and $\mu(X), \sigma(X)$ are the mean and standard deviation of the values in

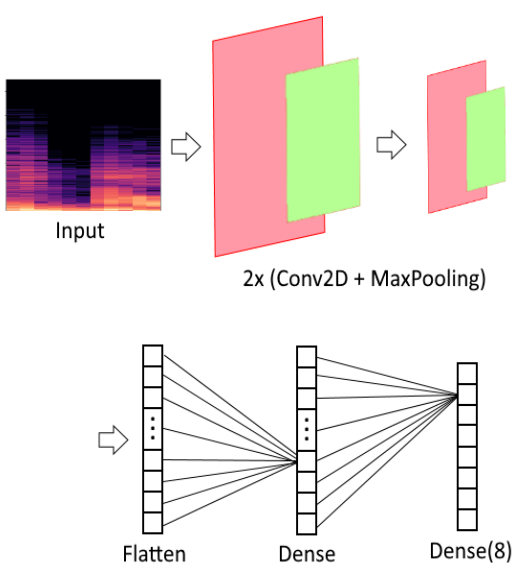

Figure 1. 2D CNN architecture.

The model architecture is described in the followings:

- 2D Convolution (16 filters, 5x5 kernel size, ReLU activation)

- $\quad$ Max Pooling (stride $=2)+$ Dropout $(0.5$ probability)

- 2D Convolution (16 filters, 5x5 kernel size, ReLU activation)

- $\quad$ Max Pooling (stride=2) + Dropout (0.5 probability)

- $\quad$ Flattening

- Dense layer (size corresponds to the mean of the flattened layer and output layer sizes)

- $\quad$ Dense (Output) layer (size corresponds to the number of classes)

Figure 1 illustrates the details of this architecture. 


\subsubsection{SVM}

Some traditional and explainable methods are used for comparison against the deep learning models.

The first such method is the Support Vector Machine. SVM constructs a hyperplane that separates the classes by maximizing the margin between the outermost points of the classes.

Since the linear separation of data is easier in higher dimensions, SVM can potentially achieve better results with higher dimensional data. A popular solution called the kernel trick is used to project the data into a higher-dimensional space. Any transformation can be used as a kernel function, some popular variants exist, such as the polynomial function or the Gaussian radial basis function. A few of these kernels were compared on both devices to find the optimal configuration.

\subsubsection{KNN}

The second traditional method is the K-nearest Neighbors (KNN) model. KNN effectively works without the necessity of a training phase: the points are classified using the $k$ nearest elements of the training set and based on a majority vote. A few of these $k$-values were compared on both devices to find the optimal value.

The implementation of the SVM, KNN models are taken from the Scikit-learn machine learning library. Both algorithms run on the respective machine's CPU.

\subsection{Low-Precision Calculations}

\subsubsection{Half-Precision Inference}

A popular method for lower bit-width weight representation is the use of 16-bit float weights. The memory representation of a 16-bit floating-point number can be seen in Figure 2. There is a higher risk of overflows and underflows with the use of 16-bits, as the represented range of numbers is significantly smaller. In practice, the use of half-precision networks with the appropriate architectural constructions has resulted in decreased memory usage with a negligible loss of accuracy (Courbariaux, Bengio, \& David, 2015).

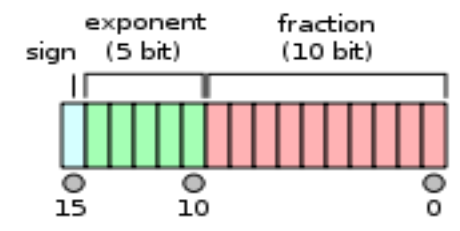

Figure 2. 16-bit float representation

There is precedent for the use of 16-bit float weights in the training phase too, but in this experiment, their use will be restricted only to the inference step. The recent bfloat 16 representation is not discussed in this paper.

Nvidia TensorRT is a library for deep learning inference and model optimization, compression. There are reports of 10-fold speedup for deep models after being optimized using the available TensorRT methods: mixed-precision conversion (16-bit float, 8-bit integer), layer fusion, parallelization, dynamic batching. The library supports the use of ONNX exported networks, thus models created in third-party deep learning frameworks such as Tensorflow and PyTorch can be imported into a TensorRT experiment.

\subsubsection{8-Bit Weight Quantization}

Weight quantization is the process of converting the weights of the neural network from 32-bit float representation to integer representation. The integer bit-width can vary, while the most popular bit-width is 8 bits, there are promising results with more aggressive 4-bit (Zhou, et al., 2018), 2-bit, or even 1-bit (binarized neural networks (Courbariaux, Hubara, Soudry, El-Yaniv, \& Bengio, 2016)) representations.

Quantized networks are predominantly used at inference, i.e.: training is performed using traditional 32-bit float weights. This experiment implements a per-tensor, symmetric quantization solution. In the case of symmetric quantization, the procedure follows the following formula:

$$
x_{q}=\operatorname{round}\left(x_{f} \cdot \frac{2^{n}-1}{\max _{x_{f}}-\min _{x_{f}}}\right)
$$


where $x_{q}, x_{f}, n$ are the quantized value, the original floating-point value and the number of bits used (here 8 ), respectively. The floating-point values are mapped to the $[-128,127] \cap \mathbb{Z}$ range. Per-tensor (also called per-layer) quantization means that all weights in a layer are scaled the same way, while in the case of per-channel quantization each channel (e.g.: convolutional kernel) has a different scale and offset.

The Neural Network Distiller (Zmora, Jacob, Zlotnik, Elharar, \& Novik, 2019) is a vast library of implemented model compression methods from scientific papers. The library is based on PyTorch. In the subfield of quantization, there is a variety of algorithms implemented.

From the PyTorch API, 3 different quantization approaches were compared:

- Post-training static quantization: The algorithm feeds batches of data through the network to learn the weight and activation distributions. This data is then used to quantize the layers.

- Dynamic quantization: It involves not just the conversion of the weights to int 8 - as it happens in all quantization variants - but also converting the activations to int8 on the fly.

- Quantization-Aware Training: Weights and activations are "fake quantized" during training: float values are rounded to mimic int 8 values, but the computations still involve floating point numbers.

The Neural Network Distiller and TensorRT solutions both implement some variant of the post-training static quantization.

\section{EVALUATION}

The dataset is randomly split into training and testing sets with a train-test ratio of $90-10 \%$.

The models are evaluated and compared based on the following metrics:

- $\quad$ Classification metric - Accuracy:

Accuracy is the proportion of samples correctly classified as positive and correctly classified as negative over the whole population. Mathematically:

- Classification metric - Precision:

$$
A c c=\frac{T P+T N}{T P+F P+T N+F N}
$$

Precision is the proportion of predicted positives that are truly positive.

- $\quad$ Classification metric - Recall:

$$
\text { Prec }=\frac{T P}{T P+F P}
$$

Recall is the proportion of true positives that are classified correctly as positive.

- $\quad$ Memory footprint:

$$
\operatorname{Rec}=\frac{T P}{T P+F N}
$$

The memory usage of a program at a specific point in time is queried by the psutil Python library.

- Inference time:

Inference time is measured using Python's time library.

\section{RESULTS}

The overall results are summarized in Tables 2, 3, 4, 5. Inference time is measured in seconds. Graphical comparisons of the model accuracies can be seen in Figures 3, 4 while memory footprint comparisons are shown in Figure 5. 


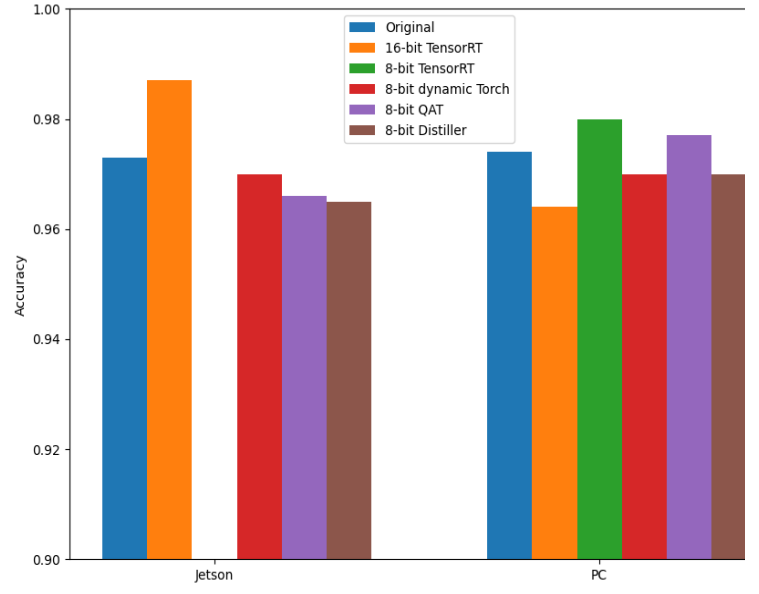

Figure 3. Accuracy of the DNN model on the PC and the Jetson

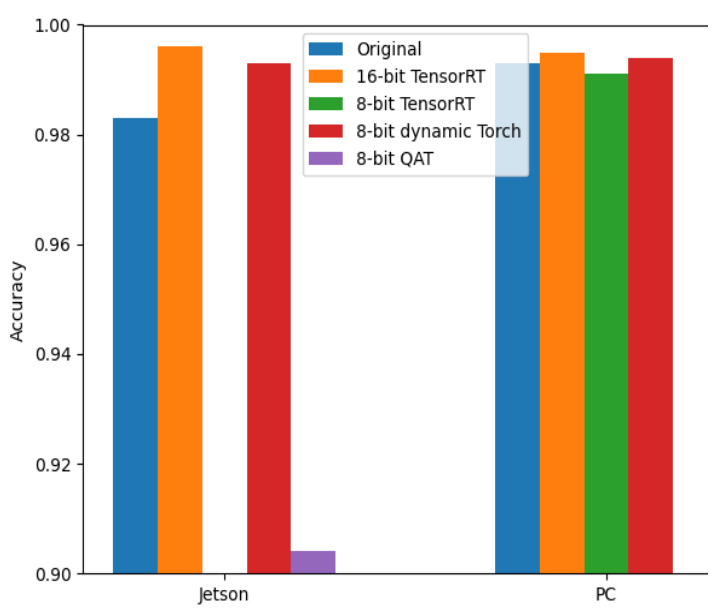

Figure 4. Accuracy of the CNN model on the PC and the Jetson

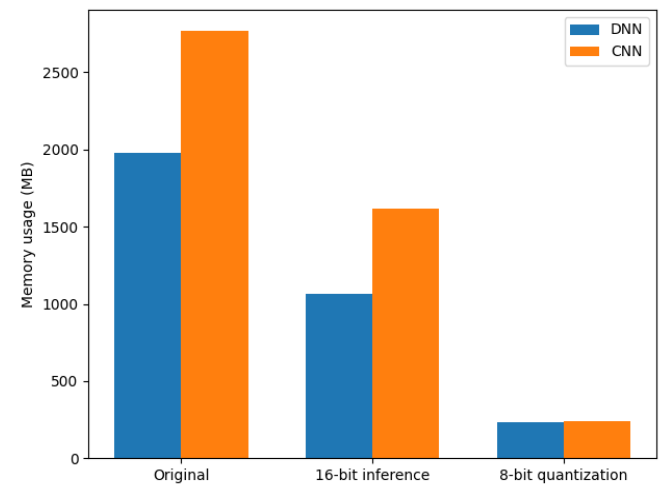

Figure 5. Comparison of model memory footprints on the Jetson

Table 2. DNN model results on the PC

\begin{tabular}{|c|c|c|c|c|}
\hline Method & Acc. & Prec. & Recall & Time \\
\hline Original & 0.974 & 0.974 & 0.974 & 0.006 \\
\hline 16-bit TensoRT & 0.964 & 0.964 & 0.964 & - \\
\hline $\begin{array}{c}\text { 8-bit post-t. } \\
\text { Torch }\end{array}$ & 0.974 & 0.974 & 0.974 & 0.004 \\
\hline 8-bit dyn. Torch & 0.970 & 0.971 & 0.970 & 0.004 \\
\hline $\begin{array}{c}\text { 8-bit QAT } \\
\text { Torch }\end{array}$ & 0.977 & 0.977 & 0.977 & 0.004 \\
\hline $\begin{array}{c}\text { 8-bit post-t. } \\
\text { Distiller }\end{array}$ & 0.970 & 0.971 & 0.970 & 0.008 \\
\hline $\begin{array}{c}\text { 4-bit post-t. } \\
\text { Distiller }\end{array}$ & 0.417 & 0.838 & 0.417 & 0.009 \\
\hline $\begin{array}{c}\text { 2-bit post-t. } \\
\text { Distiller }\end{array}$ & $<0.1$ & $<0.1$ & $<0.1$ & 0.009 \\
\hline 8-bit TensorRT & 0.980 & 0.981 & 0.980 & - \\
\hline
\end{tabular}

Table 3. CNN model results on the PC

\begin{tabular}{|c|c|c|c|c|}
\hline Method & Acc. & Prec. & Recall & Time \\
\hline Original & 0.993 & 0.993 & 0.993 & 0.067 \\
\hline 16-bit float & 0.995 & 0.995 & 0.995 & - \\
\hline $\begin{array}{c}\text { 8-bit post- } \\
\text { t.Torch }\end{array}$ & 0.595 & 0.838 & 0.595 & 0.334 \\
\hline $\begin{array}{c}\text { 8-bit dyn. } \\
\text { Torch }\end{array}$ & 0.994 & 0.994 & 0.994 & 0.129 \\
\hline $\begin{array}{c}\text { 8-bit QAT } \\
\text { Torch }\end{array}$ & 0.886 & 0.896 & 0.886 & 0.309 \\
\hline $\begin{array}{c}\text { 8-bit } \\
\text { TensorRT }\end{array}$ & 0.991 & 0.991 & 0.991 & - \\
\hline
\end{tabular}


Table 4. DNN model results on the Jetson device

\begin{tabular}{|c|c|c|c|c|}
\hline Method & Acc. & Prec. & Recall & Time \\
\hline Original & 0.973 & 0.973 & 0.973 & 1.54 \\
\hline 16-bit float & 0.987 & 0.987 & 0.987 & - \\
\hline $\begin{array}{c}\text { 8-bit post-t. } \\
\text { Torch }\end{array}$ & 0.968 & 0.968 & 0.968 & 0.269 \\
\hline 8-bit dyn. Torch & 0.970 & 0.971 & 0.970 & 0.144 \\
\hline $\begin{array}{c}\text { 8-bit QAT } \\
\text { Torch }\end{array}$ & 0.966 & 0.967 & 0.966 & 0.156 \\
\hline $\begin{array}{c}\text { 8-bit post-t. } \\
\text { Distiller }\end{array}$ & 0.965 & 0.966 & 0.965 & 0.066 \\
\hline $\begin{array}{c}\text { 4-bit post-t. } \\
\text { Distiller }\end{array}$ & 0.596 & 0.866 & 0.596 & 0.064 \\
\hline $\begin{array}{c}\text { 2-bit post-t. } \\
\text { Distiller }\end{array}$ & $<0.1$ & $<0.1$ & $<0.1$ & 0.060 \\
\hline
\end{tabular}

Table 5. CNN model results on the Jetson device

\begin{tabular}{|c|c|c|c|c|}
\hline Method & Acc. & Prec. & Recall & Time \\
\hline Original & 0.983 & 0.983 & 0.983 & 3.23 \\
\hline 16-bit float & 0.996 & 0.996 & 0.996 & - \\
\hline $\begin{array}{c}\text { 8-bit post-t. } \\
\text { Torch }\end{array}$ & 0.980 & 0.981 & 0.980 & 2.966 \\
\hline $\begin{array}{c}\text { 8-bit dyn } \\
\text { Torch }\end{array}$ & 0.993 & 0.994 & 0.993 & 96.559 \\
\hline $\begin{array}{c}\text { 8-bit QAT } \\
\text { Torch }\end{array}$ & 0.904 & 0.910 & 0.904 & 2.741 \\
\hline
\end{tabular}

\section{DISCUSSION}

The results contain some promising and interesting information. Most importantly, both the 8-bit integer quantization and the 16-bit float half-precision calculations achieve similar values in terms of the 3 classification metrics.

The methods of PyTorch are easy to use, as they are accessible through a high-level API. From the 3 presented quantization approaches, the dynamic quantization seems to be the most stable one on both the Jetson and the PC. It is even outperforming the original CNN (Convolutional Neural Network) model on the Jetson machine. To be noted, that the dynamic quantization had a significantly higher inference time on the Jetson / CNN model, for unknown reasons.

The Neural Network Distiller library is a highly useful collection of algorithms for researchers, as it contains the implementations of methods presented in a number of important papers. As it also offers the possibility to use fewer bits than 8 to represent the weights, 4- and 2-bit representations were compared as well. Although the 8-bit quantization has promising results, the smaller bit sizes produce significantly weaker and unusable models.

Considering the TensorRT methods, the results are consistently positive. However, in terms of the inference time, the amount of time that the inference needs is ambiguous, as the library performs a number of preliminary and model optimization tasks, that in total take a longer time (around 2 minutes for the Jetson, in the case of the $\mathrm{CNN}$ model). The configuration of the library is also cumbersome, and errors can frequently occur.

An important result lies in the difference in the memory footprints. The 8-bit quantized DNN model has a memory footprint of around 8.5 times smaller compared to the original model when using the PyTorch quantization. Similarly, for the CNN, the memory usage is around 11.4 times less.

The SVM seems to be unfit for this dataset, as it produces weaker results, poor predictions (Table 7). The model size is also considerably larger, 13.2 MB and the memory usage is high, $1977 \mathrm{MB}$. From the available kernels, the radial basis function (rbf) achieves the best results. The KNN model produces good quality predictions (Table 6) and has reasonable memory usage (281 MB). The running time however is higher and the model size inconveniently high $(47.7 \mathrm{MB})$, resulting in the biggest model size by far. The optimal value for $k$ proves to be 3 . 
Table 6. KNN Results

\begin{tabular}{|c|c|c|c|c|c|}
\hline $\begin{array}{c}\text { Platfor } \\
\mathbf{m}\end{array}$ & $\mathbf{k}$ & Acc. & $\begin{array}{c}\text { Prec } \\
\cdot\end{array}$ & $\begin{array}{c}\text { Recal } \\
\mathbf{l}\end{array}$ & $\begin{array}{c}\text { Tim } \\
\mathbf{e}\end{array}$ \\
\hline PC & 3 & $\begin{array}{c}0.88 \\
1\end{array}$ & $\begin{array}{c}0.86 \\
5\end{array}$ & 0.881 & 0.01 \\
0
\end{tabular}

Table 7. SVM Results

\begin{tabular}{|c|c|c|c|c|c|}
\hline $\begin{array}{c}\text { Platfor } \\
\mathbf{m}\end{array}$ & kernel & Acc. & $\begin{array}{c}\text { Prec } \\
\cdot\end{array}$ & $\begin{array}{c}\text { Recal } \\
\mathbf{l}\end{array}$ & $\begin{array}{c}\text { Tim } \\
\mathbf{e}\end{array}$ \\
\hline PC & linear & $\begin{array}{c}0.76 \\
2\end{array}$ & $\begin{array}{c}0.77 \\
5\end{array}$ & 0.762 & $\begin{array}{c}0.01 \\
4\end{array}$ \\
\hline PC & poly & $\begin{array}{c}0.58 \\
9\end{array}$ & $\begin{array}{c}0.81 \\
2\end{array}$ & 0.589 & $\begin{array}{c}0.02 \\
0\end{array}$ \\
\hline PC & rbf & $\begin{array}{c}0.82 \\
8\end{array}$ & $\begin{array}{c}0.84 \\
4\end{array}$ & 0.828 & $\begin{array}{c}0.04 \\
6\end{array}$ \\
\hline PC & sigmoi & $\begin{array}{c}0.60 \\
\text { d }\end{array}$ & $\begin{array}{c}0.63 \\
0\end{array}$ & 0.609 & $\begin{array}{c}0.01 \\
9\end{array}$ \\
\hline Jetson & linear & $\begin{array}{c}0.76 \\
2\end{array}$ & $\begin{array}{c}0.77 \\
5\end{array}$ & 0.762 & $\begin{array}{c}0.16 \\
5\end{array}$ \\
\hline Jetson & poly & $\begin{array}{c}0.58 \\
9\end{array}$ & $\begin{array}{c}0.81 \\
2\end{array}$ & 0.589 & $\begin{array}{c}0.16 \\
1\end{array}$ \\
\hline Jetson & rbf & $\begin{array}{c}0.82 \\
8\end{array}$ & $\begin{array}{c}0.84 \\
4\end{array}$ & 0.828 & $\begin{array}{c}0.17 \\
9\end{array}$ \\
\hline Jetson & sigmoi & $\begin{array}{c}0.60 \\
\text { d }\end{array}$ & $\begin{array}{c}0.63 \\
0\end{array}$ & 0.609 & $\begin{array}{c}0.17 \\
5\end{array}$ \\
\hline
\end{tabular}

In conclusion, the favorable results indicate that the use of model compression techniques could further advance the field of IoMT (Internet of Medical Things) and allow the implementation of medical classification problems on IoT devices. As future work, the analysis of further model compression methods like structured pruning is planned. The goal is to find compressed models that achieve both a significant decrease in memory usage and inference time.

\section{REFERENCES}

Acharya, J., \& Basu, A. (2020, 4). Deep Neural Network for Respiratory Sound Classification in Wearable Devices Enabled by Patient Specific Model Tuning. IEEE Transactions on Biomedical Circuits and Systems (TBioCAS. doi:10.1109/tbcas.2020.2981172

Courbariaux, M., Bengio, Y., \& David, J.-P. (2015, 12). Training Deep Neural Networks with Low Precision Multiplications. Accepted as a workshop contribution to ICLR 2015.

Courbariaux, M., Hubara, I., Soudry, D., El-Yaniv, R., \& Bengio, Y. (2016, 2). Binarized Neural Networks: Training Deep Neural Networks with Weights and Activations Constrained to +1 or -1 . Advances in Neural Information Processing Systems 29 (NIPS 2016).

Hadidi, R., Cao, J., Xie, Y., Asgari, B., Krishna, T., \& Kim, H. (2019, 11). Characterizing the Deployment of Deep Neural Networks on Commercial Edge Devices. 2019 IEEE International Symposium on Workload Characterization (IISWC) (pp. 35-48). IEEE. doi:10.1109/iiswc47752.2019.9041955

Jain, A., Bhattacharya, S., Masuda, M., Sharma, V., \& Wang, Y. (2020, 6). Efficient Execution of Quantized Deep Learning Models: A Compiler Approach. Retrieved from https://arxiv.org/abs/2006.10226.

Mermelstein, P. (1976). Distance Measures for Speech Recognition, Psychological and Instrumental. Pattern recognition and artificial intelligence, 116, 374-388.

Pál, T., Molnár, B., \& Tarcsi, Á. (2020, 10). Lightweight, Length Invariant Models and Dimensionality Reduction in Respiratory Disease Detection. Annales Universitatis Scientiarum Budapestinensis de Rolando Eötvös Nominatae. Sectio Computatorica. Submitted, unpublished.

Paszke, A., Gross, S., Massa, F., Lerer, A., Bradbury, J., Chanan, G., . . Chintala, S. (2019). Pytorch: An Imperative Style, High-performance Deep Learning Library. In H. M. Wallach, H. Larochelle, A. Beygelzimer, F. d'Alché-Buc, E. B. Fox, \& R. Garnett (Ed.), Advances in Neural Information Processing Systems 32: Annual Conference on Neural Information Processing Systems 2019, NeurIPS 2019, December 8-14, 2019, Vancouver, BC, Canada, (pp. 8024-8035). 
Quantized Neural Network Package. (n.d.). Quantized Neural Network Package. Retrieved from https://github.com/pytorch/QNNPACK

Rocha, B. M., Filos, D., Mendes, L., Vogiatzis, I., Perantoni, E., Kaimakamis, E., . . Maglaveras, N. (2018). A Respiratory Sound Database for the Development of Automated Classification. In N. Maglaveras, I. Chouvarda, \& de Carvalho P. (Eds.), Precision Medicine Powered by pHealth and Connected Health. ICBHI 2017. IFMBE Proceedings (Vol. 66, pp. 33-37). Springer, Singapore. doi:10.1007/978-981-10-7419-6_6

Yang, Y., Chen, A., Chen, X., Ji, J., Chen, Z., \& Dai, Y. (2018, 5). Deploy Large-scale Deep Neural Networks in Resource Constrained Iot Devices with Local Quantization Region. Retrieved from https://arxiv.org/abs/1805.09473.

Zhou, S., Wu, Y., Ni, Z., Zhou, X., Wen, H., \& Zou, Y. (2018, 6). Dorefa-net: Training Low Bitwidth Convolutional Neural Networks with Low Bitwidth Gradients. Retrieved from https://arxiv.org/abs/1606.06160.

Zmora, N., Jacob, G., Zlotnik, L., Elharar, B., \& Novik, G. (2019, 10). Neural Network Distiller: A Python Package For DNN Compression Research. Retrieved from https://arxiv.org/abs/1910.12232 